\title{
HABLANDO EN PROSA SIN SABERLO: REFLEXIONES SOBRE LA ARTICULACIÓN TERRITORIAL DEL ESTADO EN LA CONSTITUCIÓN ESPAÑOLA DE 1978
}

\author{
JAVIER PÉREZ ROYO \\ Catedrático de Derecho Constitucional \\ Universidad del Sevilla
}





\title{
HABLANDO EN PROSA SIN SABERLO: REFLEXIONES SOBRE LA ARTICULACIÓN TERRITORIAL DEL ESTADO EN LA CONSTITUCIÓN ESPAÑOLA DE 1978*
}

\author{
POR \\ JAVIER PÉREZ ROYO \\ Catedrático de Derecho Constitucional \\ Universidad del Sevilla
}

La singularidad más llamativa de nuestra historia constitucional reside en que el problema más importante para la definición del Estado, la articulación territorial del mismo, ha brillado por su ausencia en los diferentes procesos constituyentes a través de los cuales se ha ido imponiendo trabajosamente el Estado Constitucional en España. La sociedad española, aunque parezca increíble, no ha protagonizado nunca en sede constituyente un debate sobre la estructura de su Estado. Se ha constituido políticamente sin interrogarse nunca de manera expresa sobre cómo deberían integrarse las diferentes partes del territorio en un Estado común.

En las Constituciones del XIX, con la excepción, obviamente, de la Constitución Federal de la Primera República, el problema de la estructura del Estado ni siquiera se insinúa. El Estado unitario y cen-

* Ponencia presentada al VII Congreso Iberoamericano de Derecho Constitucional, México, 12-15 de febrero de 2002. 
tralista se acepta como algo evidente, que no es susceptible casi de ser sometido a discusión.

Es verdad que, como puso de manifiesto Tomás y Valiente en su estudio sobre la génesis de la primera de nuestras Constituciones, la Constitución de Cádiz de 1812, en el debate preconstituyente de dicha Constitución sí se planteó expresamente el problema de la estructura del nuevo Estado que se pretendía constituir y se exploraron alternativas distintas a las del Estado unitario. Pero no lo es menos, que tal debate preconstituyente desaparece por completo en el momento final de la fase preconstituyente, de tal manera que en el texto del proyecto de Constitución que se hace público y que sirve de base a los debates constituyentes de las Cortes de Cádiz no queda huella del mismo (Francisco Tomás y Valiente, "Génesis de la Constitución de 1812. I. De muchas Leyes Fundamentales a una sola Constitución", en Anuario de Historia del Derecho Español, tomo LXV, Madrid, 1995).

Quiere decirse, pues, que la posibilidad misma del debate sobre la estructura del Estado en el momento constituyente originario del Estado español no llegó a pasar de ser eso, una mera posibilidad. Posibilidad de la que incluso nos hemos empezado a enterar muy recientemente, pues únicamente tras la entrada en vigor de la Constitución de 1978 y la transformación del Estado español unitario y centralista en el Estado de las Autonomías, ha empezado la investigación histórica a rastrear los posibles orígenes de esta nueva forma de Estado. El debate preconstituyente de Cádiz, desenterrado por Tomás y Valiente, es como si no hubiera tenido lugar. Nadie ha sabido a lo largo de estos dos últimos siglos que tal debate se había producido o, por lo menos, nadie había llamado públicamente la atención sobre el mismo.

El golpe de mano mediante el cual se suprimió la posibilidad de que en el debate constituyente de Cádiz se discutiera sobre la estructura territorial del Estado español tuvo consecuencias de largo alcance. La Constitución de Cádiz estuvo muy poco tiempo en vigor, pero el debate constituyente de Cádiz fijó los términos del debate constitucional en España a lo largo del siglo. Lo que no se planteó en términos constituyentes en Cádiz no vuelve a plantearse en tales términos a lo largo del siglo.

El problema, obviamente, no desaparece por completo, ya que forma parte de nuestra Constitución material, pero las manifestaciones del mismo no llegarán nunca a los textos constitucionales, sino que se quedarán en un nivel infraconstitucional. En ninguna de las Constituciones posteriores a la de Cádiz a lo largo del siglo, ni siquiera en fase preconstituyente que sepamos, se insinúa la existencia de 
un problema de esta naturaleza. La única excepción, como antes se indicó, fue la Constitución Federal de la Primera República, que fue más la expresión del momento final de descomposición de la experiencia constitucional puesta en marcha por la Revolución de 1868, que una experiencia constituyente propiamente dicha. En el constitucionalismo español del XIX la experiencia federal republicana de 1873 es un caso claro de excepción que confirma la regla.

Si el mismo jurista persa, al que Pedro Cruz Villalón puso a leer la Constitución de 1978 para intentar entender cuál era la estructura del Estado español definida en dicha Constitución, leyera las Constituciones españolas del siglo XIX, llegaría a la conclusión de que el problema de la estructura territorial del Estado no había existido en España en todo el siglo. (Pedro Cruz Villalón. "La estructura del Estado o la curiosidad del jurista persa", en La curiosidad del jurista persa, y otros estudios sobre la Constitución, Centro de Estudios Constitucionales, Madrid, 1999). En ningún momento llegó a plantearse en sede constituyente que el Estado pudiera estructurarse territorialmente de manera descentralizada.

Completamente distinta parece ser, a primera vista, la experiencia constitucional española del siglo $X X$. No sólo en el sentido de que la articulación territorial del Estado se plantea expresamente en los dos procesos constituyentes que ha protagonizado la sociedad española en dicho siglo, sino que, además, en ambos se plantea expresamente como el problema central del debate constitucional. Cuando se lee el texto del Anteproyecto de Constitución de la República española que eleva al Gobierno la Comisión Jurídica Asesora de 1931 o la valoración general del Proyecto de Constitución que se hace por los portavoces de los distintos grupos parlamentarios en las Cortes constituyentes de 1978, parece que ese problema de la articulación territorial del Estado es el problema constitucional decisivo y que en torno a él, en consecuencia, va a girar el debate constituyente.

$Y$ sin embargo, en ninguno de ambos procesos constituyentes va a ser así. Políticamente nadie discutía ni discute que era efectivamente así. Más en el proceso constituyente de 1978 que en el de 1931, aunque se expresara de manera más urgente en éste que en aquél. Pero jurídicamente ni en 1931 ni en 1978 va a ser capaz de afrontar la sociedad española el debate en términos generales. En la sociedad española no ha llegado a madurar hasta la fecha una respuesta política al problema de la estructura del Estado que pudiera ser formalizada jurídicamente. En los dos momentos constituyentes del siglo $X X$, en los que se suponía que teníamos que hablar de todos los problemas de articulación constitucional de nuestro Estado, no hemos sido capaces de hablar abiertamente de cómo tenía que articularse territorialmente el Estado 
español. Hemos sido capaces de hablar abiertamente y en términos generales de todos los problemas, menos del más importante, que, precisamente por eso, todavía no está resuelto. No hemos sido capaces de definir constitucionalmente la estructura del Estado y hemos remitido siempre el problema constitucional al proceso político que tendría que desarrollarse a partir de la entrada en vigor de la Constitución. Nuestros dos procesos constituyentes del siglo XX han devaluado constitucionalmente la respuesta a la estructura territorial del Estado, que no ha tenido, por eso, una respuesta constitucional en sede constituyente, sino que en ambos casos ha tenido que encontrar una respuesta una vez finalizado el proceso constituyente formal a través de normas no constitucionales si bien dictadas con base en la Constitución. La estructura del Estado no se ha resuelto nunca en España en la Constitución sino con base en la Constitución.

La Comisión Jurídica Asesora de 1931 marcó la pauta. "Materia primera de nuestra preocupación fue la referente a la estructura de España en régimen unitario o federal'). Éstas son prácticamente las primeras palabras de su Dictamen de presentación del Anteproyecto de Constitución, ya que las que las preceden son puramente retóricas. Las primeras palabras en las que se aborda materia constitucional son éstas. En consecuencia, parecería lógico esperar que a continuación se abordara frontalmente el tema y se hiciera una propuesta coherente con el carácter de "primera preocupación» con que se la había definido.

Y sin embargo, no va a ser así. "Dividida hoy la opinión entre uno y otro sistema", continúa la Comisión, "fácil hubiera șido pronunciarse por cualquiera, ya que en apoyo de ambos se dan razones de gran peso dogmático e histórico. No obstante, la Comisión ha entendido preferible -coincidiendo con opiniones muy valiosas- no teorizar sobre tema tan grave, sino apoyarse en la innegable realidad de hoy y abrir camino a la posible realidad de mañana».

La renuncia a enfrentarse directamente con el problema no puede reconocerse de manera más clara. La subordinación expresa de la Constitución formal a la Constitución material, que las palabras que hemos transcrito en cursiva traducen, es llamativa en un proceso constituyente democrático, en el que al mismo tiempo se va a afirmar por primera vez en nuestra historia la Constitución como norma jurídica y se van a establecer garantías de su superioridad sobre todas las demás normas del ordenamiento.

Por si cabía alguna duda de qué significaban dichas palabras, el párrafo siguiente del texto de la Comisión Jurídica Asesora se encarga de despejarla: "Las provincias han adquirido, en el curso de un 
siglo, personalidad y relieve que nadie puede desconocer; y en la mayor parte del territorio nacional nadie protesta contra esta organización ni reclama otra. Hubiera sido, pues, arbitrario trazar sobre el papel una República federal que, por lo visto, no apetece a la generalidad del pueblo a quien había de serle impuesta. Mas con igual claridad, en otras regiones españolas han surgido o apuntan anhelos de personalidad autónoma, en términos tan vivos, con razones tan fuertes y con apasionamiento tan considerable, que el cerrar los caminos a su expansión sería, sobre una injusta negación del sentido de libertad, una insigne torpeza política. He aquí por qué la Comisión ha preferido, en vez de inventar un federalismo uniforme y teórico, facilitar la formación de entidades que, para alcanzar una autonomía mayor o menor, habrán de encontrar como arranque su propio deseo".

No teorizar, es decir, no constitucionalizar la respuesta al problema, sino limitarse simplemente a dar cauce a la apertura de un proceso histórico a través del cual pueda expresarse de manera inmediata la "innegable realidad de hoy" y se abra un camino "a la posible realidad de mañanan. Será la Constitución material territorial de España la que tendrá que irse abriendo camino en el marco diseñado en la Constitución, sin intentar su definición en sede constituyente.

Aunque hubo varios votos particulares en el sentido de abordar el asunto como "un problema total de la estructura de España, que ha de acometerse con valentía» y no resolverlo mediante "un compromiso" (Francisco Romero Otazo, Manuel Pedroso, Alfonso García Valdecasas, Antonio de Luna), o que proponían directamente la definición federal de la República (Nicolás Alcalá Espinosa y Javier Elola), la mayoría en el seno de la Comisión Jurídica Asesora a favor del Dictamen fue muy amplia.

Con el dictamen de la Comisión, en la que, como subrayaría el Presidente de la misma, Jiménez de Asúa, en su Discurso de presentación del proyecto de Constitución al pleno de las Cortes Constituyentes, "habían estado representados todos los grupos de la Cámara", quedarían fijados los términos del debate constituyente. Las Cortes constituyentes no sólo harían suya básicamente la propuesta de articulación territorial del Estado contenida en el Anteproyecto en el momento de la decisión, sino que, además, como consecuencia de ello, reduciría en la fase de deliberación la discusión del problema de la estructura del Estado a la "cuestión catalana" casi exclusivamente.

Las inconsecuencias constitucionales que de dicha manera de proceder se derivarían no eran de poca importancia. Dos de los órganos constitucionales más importantes del Estado Constitucional de- 
mocrático quedarían imperfecta y, por tanto, disfuncionalmente constituidos como consecuencia de esta renuncia a constitucionalizar la estructura del Estado. Y quedarían mal constituidos en direcciones opuestas. Uno por defecto y el otro por exceso. Las Cortes serían configuradas como un Parlamento unicameral, no contemplándose, en consecuencia, ningún órgano en el que las Regiones autónomas que se constituyeran pudieran participar en la formación de la voluntad del Estado. El Tribunal de Garantías Constitucionales, por el contrario, sería configurado como un órgano compuesto, entre otros, por "un representante por cada una de las Regiones españolas", independientemente de que se hubieran constituido o no en Regiones autónomas. Lo que faltaba en la definición del momento político del Estado, sobraba en la definición del momento jurídico. Es lo que suele ocurrir cuando falta claridad en los fundamentos y se renuncia a "teorizar".

No cabe desconocer, sin embargo, que con base en el texto constitucional de 1931 se inició un proceso descentralizador del Estado que estaba avanzando decididamente en el momento en que se produjo la sublevación militar que desembocó en la Guerra Civil. Nunca podremos saber cuál habría sido la estructura resultante del desarrollo de dicho proceso, pero no parece aventurado afirmar que hubiera conducido a un resultado final no muy diferente del que se ha alcanzado con base en la Constitución de 1978. Pero no adelantemos conclusiones y sigamos el orden.

Esta manera de enfrentarse con el problema de la estructura territorial del Estado parecia que iba a ser corregida en el proceso constituyente de 1977-78. En esta ocasión parecía que el constituyente sí estaba dispuesto inicialmente a "teorizar", esto es, a resolver constitucionalmente el problema y no remitir su solución al proceso político posterior a la entrada en vigor de la Constitución, es decir, a la imposición en la práctica de la Constitución material territorial de España.

En efecto, si se examina el Anteproyecto de Constitución elaborado por la Ponencia elegida en el seno de la Comisión Constitucional del Congreso de los Diputados y publicado en el Boletín Oficial de las Cortes el 5 de enero de 1978, se comprueba inmediatamente que la perspectiva en la que se sitúa la Ponencia es completamente distinta a aquella en la que se situó la Comisión Jurídica Asesora en 1931. El texto del Anteproyecto de Constitución contiene una definición acabada de la estructura del Estado, no limitándose a establecer un cauce para la apertura de un proceso histórico en el que dicha estructura vaya definiéndose. 
Esta voluntad de resolver el problema en el propio texto constitucional se pone de manifiesto desde la formulación misma de la decisión política constitucionalmente conformadora de la estructura del Estado en el artículo 2: "La Constitución se fundamenta en la unidad de España y en la solidaridad entre sus pueblos y reconoce el derecho a la autonomía de las nacionalidades y regiones que la integran". La unidad como principio político del Estado, la autonomía como derecho de las nacionalidades y regiones que lo integran. Ejercicio del derecho a la autonomía condicionado por el principio de unidad, pero también realización efectiva del principio de unidad a través del ejercicio del derecho a la autonomía. Ésta era la decisión política que tenía que ser articulada posteriormente en el Título correspondiente del Anteproyecto.

Coherentemente con esta decisión, la Ponencia regula con precisión las condiciones de ejercicio del derecho a la autonomía que había sido puesto uniformemente a disposición de los titulares del mismo. La calificación de éstos como nacionalidades y regiones no entrañaba en principio ninguna diferenciación en lo que a las condiciones de ejercicio del derecho a la autonomía se refiere. De ahí que el Anteproyecto se enfrentara con el problema como un problema de la estructura del Estado y no como un problema de determinados territorios del mismo en su relación con el Estado.

A tal efecto, y apartándose de la orientación constitucional republicana del 31, la Ponencia dedicaba el Título VIII única y exclusivamente a los "Territorios Autónomos", diferenciando de manera inequívoca la autonomía de las nacionalidades y regiones de la autonomía de otros entes territoriales, como los municipios y provincias, que se incluian en el Título $\mathrm{V}$.

Las condiciones de ejercicio del derecho a la autonomía eran idénticas para todos los titulares del mismo en las cinco fases de ejercicio de dicho derecho: iniciativa del proceso autonómico, elaboración del Estatuto de Autonomía, organización política del territorio autónomo, distribución de competencias entre el Estado y el territorio autónomo y financiación del territorio autónomo.

La iniciativa del proceso autonómico la tendrían que ejercer las dos terceras partes de los municipios de cada una de las provincias que desearan constituirse en territorio autónomo. A continuación se constituiría una asamblea integrada por los parlamentarios, diputados y senadores, elegidos en las provincias, a fin de redactar el proyecto de Estatuto de Autonomía, que sería negociado posteriormente por una delegación de dicha asamblea con la Comisión Constitucional del 
Congreso de los Diputados. La organización del territorio autónomo consistiría en una Asamblea legislativa elegida por sufragio universal y un Presidente y Consejo de Gobierno elegido por ella y responsable políticamente ante ella. El sistema de distribución de competencias era el mismo para todos los territorios, limitándose el Anteproyecto a la fijación de un listado de materias reservadas a la competencia del Estado, permitiendo que los territorios autónomos a través de sus Estatutos de Autonomía asumieran competencias en todas las materias no reservadas al Estado. Por último, la financiación era regulada en términos idénticos para todos los territorios autónomos.

Se configuraba, por tanto, un Estado íntegramente descentralizado en territorios autónomos, que tendrían todos la misma naturaleza, la misma organización política, el mismo nivel competencial y el mismo sistema de financiación. El carácter inequívocamente político de todas las unidades de descentralización del Estado resultaba indiscutible y se manifestaba en todas las fases de ejercicio del derecho a la autonomía. La unidad política del Estado se canalizaba a través del reconocimiento de un derecho, pero de un derecho que no era más que un cauce para el ejercicio de un poder de naturaleza política. Ésta era la propuesta constitucional sobre la estructura del Estado del primer Anteproyecto de Constitución. No se contemplaba ninguna excepción en el ejercicio del derecho. De ahí que la regulación íntegra de la estructura del Estado figurara en el Título VIII de la Constitución, sin remisiones a Disposiciones Adicionales o Transitorias.

Como complemento de esta regulación uniforme del ejercicio del derecho a la autonomía, el Anteproyecto diseñaba un Senado compuesto de representantes de los distintos Territorios Autónomos que integran España, correspondiendo la elección de los senadores a las Asambleas legislativas de dichos Territorios (art. 60).

Como puede verse, el Anteproyecto "teorizaba", es decir, daba una respuesta constitucional global a la estructura del Estado en términos de decisión político-constitucional y en términos de desarrollo normativo de dicha decisión político-constitucional. Las bases para la discusión en sede constituyente del problema de la articulación territorial del Estado estaban sentadas. Parecía que esta vez la sociedad española sí iba a ser capaz de enfrentarse abiertamente y de intentar encontrar una respuesta a través de una discusión pública al problema de su articulación territorial.

Sin embargo, no va a ser así. Ya en la reelaboración del Anteproyecto de la Constitución por parte de la Ponencia, a partir de los votos particulares de los propios ponentes y de las enmiendas de los 
diputados, se va a renunciar, como se hizo en 1931, a la "teorización" y se va a reproducir en buena medida una respuesta del mismo estilo a la que entonces se dio al problema.

El Anteproyecto de Constitución que se publica en el Boletín Oficial de las Cortes el 17 de abril, con base en el cual se van a celebrar los debates constituyentes, se diferencia de manera radical del Anteproyecto del 5 de enero.

Lo único que se mantiene es la decisión política constitucionalmente conformadora del artículo 2, si bien redactada en unos términos que, jurídicamente, no dicen casi nada, pero que políticamente dicen casi todo: "La Constitución se fundamenta en la unidad de España como patria común e indivisible de todos los españoles y reconoce el derecho a la autonomía de las nacionalidades y regiones que integran la indisoluble unidad de la nación española». Lo que el 5 de enero era un reconocimiento sin reservas del derecho a la autonomía, se rodea el 17 de abril de todas las reservas imaginables.

Estas reservas se van a traducir en una configuración constitucional de la estructura del Estado completamente distinta de la contemplada en el Anteproyecto inicial de 5 de enero de 1978.

En primer lugar, el Anteproyecto de 17 de abril incluye en el Título VIII, que significativamente pasa a denominarse "De la organización territorial del Estado" en lugar de "De los Territorios Autónomos», la regulación de los Municipios y Provincias junto a las Comunidades Autónomas. La separación en el Anteproyecto inicial, que pretendía subrayar que la naturaleza de la autonomía de las nacionalidades y regiones era distinta de la autonomía de los Municipios y Provincias, queda cancelada en esta primera revisión del texto constitucional.

Dicha cancelación no es inocente, sino que tiene como finalidad rebajar el alcance de la autonomía de las Comunidades Autónomas y asemejarla a la autonomía de Municipios y Provincias. Si en el primer Anteproyecto la intervención de los Municipios y Provincias en el ejercicio del derecho a la autonomía de las nacionalidades y regiones se limitaba a la primera fase del mismo, la iniciativa del proceso autonómico, desapareciendo por completo después, en el Anteproyecto de 17 de abril, la presencia de los Municipios y Provincias se va a prolongar en la segunda fase, en la elaboración del Estatuto de Autonomía, que será redactado ahora por una Asamblea integrada no solamente por los parlamentarios estatales elegidos en las provincias que se van a constituir en Comunidad Autónoma, sino además por los 
diputados provinciales, es decir, por representantes no elegidos directamente por los ciudadanos, sino indirectamente a través de las elecciones municipales. El Proyecto de Estatuto aprobado por esa Asamblea mixta no se negocia después con la Comisión Constitucional del Congreso de los Diputados, sino que se remite a las Cortes Generales para su aprobación como ley orgánica. El cambio, como puede verse, no puede ser más significativo. Las Comunidades Autónomas previstas en el Anteproyecto de 17 de abril no ejercen una autonomía de naturaleza política, sino que se limitan a proponer al Estado un proyecto de Estatuto, para que sea éste el que decida. Las Comunidades Autónomas pierden por completo el control sobre el proceso de su propia constitución como tales.

En lo que a la organización política de las Comunidades Autónomas se refiere, el Anteproyecto se limita a indicar que el Estatuto de Autonomía deberá contener "la denominación, organización y sede de las instituciones autónomas propias» (art. 139.1.c), suprimiendo cualquier referencia a la Asamblea legislativa y al Presidente y Consejo de Gobierno contenidos en el Anteproyecto inicial.

En lo relativo a la distribución de competencias entre el Estado y las Comunidades Autónomas la redacción del anterior artículo 138, ahora 141, continúa siendo la misma, pero su alcance es completamente distinto. Si las Comunidades Autónomas que se constituyan no se sabe si van a tener Asamblea legislativa y Gobierno propio, es imposible saber qué tipo de competencias van a poder asumir. Sin la delimitación de la organización política de las Comunidades Autónomas el precepto de distribución competencial entre ellas y el Estado se convierte en un precepto vacío.

$Y$ esto es todo lo que contiene el Título VIII en este segundo Anteproyecto. Frente a la constitucionalización de la estructura del Estado en el primer Anteproyecto, en este segundo se produce una desconstitucionalización de la misma. No se sabe cuál va a ser la naturaleza de las Comunidades Autónomas que se constituyan, no se sabe cuál va a ser su organización institucional y no se sabe cuál va a ser la distribución competencial entre el Estado y las Comunidades Autónomas. El único momento del ejercicio del derecho a la autonomía que se define con precisión en el Anteproyecto, el de la elaboración del Estatuto de Autonomía, se sustrae a la decisión de la Comunidad Autónoma, que queda reducida a la posición de elaboradora de un proyecto sobre cuyo destino final pierde por completo el control. Las Comunidades Autónomas serán lo que el Estado quiera que sean. Llamar a eso ejercicio del derecho a la autonomía es difícil de aceptar. 
Esta desconstitucionalización de la estructura del Estado va a tener su reflejo en la incapacidad del Anteproyecto de ofrecer ninguna propuesta sobre la composición del Senado. Aunque parezca increíble, el texto remitido por la Ponencia para que se iniciara el debate constituyente carece de propuesta sobre la composición de la Segunda Cámara.

Esto es todo lo que se contiene en el Anteproyecto de Constitución stricto sensu, pero no es todo lo que el Anteproyecto contiene respecto de la estructura del Estado, ya que, además del Título VIII, el Anteproyecto contiene una muy extensa Disposición Adicional en la que se regula también el ejercicio del derecho a la autonomía.

Pero no el ejercicio del derecho a la autonomía para todas las nacionalidades y regiones, sino únicamente para algunas de ellas. Frente a la constitucionalización uniforme de las condiciones de ejercicio del derecho a la autonomía del Anteproyecto inicial, en el segundo se va a optar por una constitucionalización diferenciada.

Las Comunidades Autónomas «donde hubieran sido aprobados legalmente estatutos de autonomía mediante sufragio universal» (apartado 4) podrán constituirse en Comunidades Autónomas en los términos en que estaba previsto el ejercicio del derecho a la autonomía en el Anteproyecto inicial de Constitución. Lo que en éste aparecía como regulación uniforme para todas, queda reducido en el segundo Anteproyecto a regulación sólo para algunas.

Esta diferenciación entre unas Comunidades Autónomas y otras no se impone, sin embargo, de manera taxativa, sino que se admite la posibilidad de que las Comunidades Autónomas, en las que no se hubieran aprobado estatutos de autonomía por sufragio universal en el pasado, también pudieran acceder al ejercicio del derecho a la autonomía en las mismas condiciones que aquellas en las que sí se habia aprobado, si el ejercicio de la iniciativa del proceso autonómico se hacía con mayorías muy reforzadas y con un referéndum de ratificación de dicha iniciativa en el que se exigía la mayoría absoluta del censo electoral de cada una de las provincias que quisieran constituirse en comunidad autónoma (apartado 5).

El segundo Anteproyecto abandona, por tanto, la perspectiva de la constitucionalización de la estructura del Estado, para pasar a una constitucionalización limitada de dicha estructura, en la que únicamente queda definida constitucionalmente la posición de aquellas Comunidades Autónomas en las que se hubieran aprobado en el pasado por sufragio universal Estatutos de Autonomía, dejando en un limbo constitucional a las demás. 
La perspectiva del segundo Anteproyecto de Constitución es en realidad la perspectiva de la Comisión Jurídica Asesora de 1931. Se da una respuesta inmediata al ejercicio del derecho a la autonomía para Cataluña, País Vasco y Galicia, esto es, a "la innegable realidad de hoy", y se remite "la posible realidad de mañana", esto es, la respuesta para las demás, al proceso histórico posterior a la entrada en vigor de la Constitución, aunque abriendo una vía para que estas últimas pudieran incorporarse al grupo de las primeras.

La reelaboración del texto por la Ponencia tuvo consecuencias extraordinarias para el debate constituyente. De la misma manera que la posición de la Comisión Jurídica Asesora en 1931 fijó los términos del debate constituyente republicano, la posición de la Ponencia en el texto que acabó enviando como Anteproyecto para su discusión en sede constituyente fijó también los términos del debate. En realidad, la regulación de la estructura del Estado que se contiene en el texto definitivamente aprobado de la Constitución es la misma del segundo Anteproyecto de Constitución, aunque reorganizada de manera distinta. La casi totalidad de la Disposición Adicional del Anteproyecto se incorporará al Título VIII definitivo, quedando una pequeña parte de la misma incluida en la Disposición Transitoria 2.?. Pero materialmente la respuesta al problema de la estructura del Estado es la misma. Todos los elementos esenciales de la respuesta constitucional a la estructura del Estado estaban ya en el Anteproyecto de Constitución de 17 de abril y sobre ellos va a girar el debate constituyente. Frente a la voluntad de constitucionalización del Anteproyecto de 5 de enero se acabará imponiendo una voluntad de desconstitucionalización en el del 17 de abril, que será la que definitivamente hagan suya las Cortes Constituyentes.

La posibilidad de que hubiera un debate en sede constituyente sobre la estructura territorial del Estado, abierta con el primer Anteproyecto de Constitución, se perdió con el segundo. La perspectiva general desapareció de manera definitiva. Se procedía de nuevo a una devaluación constitucional de la estruetura del Estado, que tendría que ser definida a partir de y con base en la Constitución, pero que no quedaba definida en el texto constitucional.

Quiere decirse, pues, que la definición de la estructura del Estado se remitía al proceso histórico que se abriera inmediatamente después de la entrada en vigor de la Constitución. El poder constituyente remitía al poder estatuyente la concreción de la estructura descentralizada del Estado que la Constitución posibilitaba pero no definía. De la interpretación que se acabara imponiendo de la Constitución dependería la estructura final del Estado. 
En principio, las alternativas teóricas que la Constitución ofrecía eran varias, pero las históricamente posibles eran solamente dos: la interpretación en clave nacionalista o diferenciadora del derecho a la autonomía y su ejercicio, o la interpretación en clave homogeneizadora, como un problema general de la estuctura del Estado, que debería territorializarse por completo y de manera uniforme o, si se prefiere, simétrica.

La interpretación diferenciadora fue la primera que se ensayó inmediatamente después de la entrada en vigor de la Constitución. Tal interpretación suponía consagrar orgánica y funcionalmente la distinción entre nacionalidades y regiones como titulares del derecho a la autonomía reconocido en el artículo 2 de la Constitución. Las nacionalidades ejercerian el derecho a la autonomía de manera distinta a como lo harian las regiones. Serían las únicas que se constituirían como expresión de un poder político propio en los términos en que se contemplaba el ejercicio del derecho a la autonomía en el primer Anteproyecto de Constitución.

En efecto, las nacionalidades se verían eximidas de pasar por la fase de la iniciativa del proceso autonómico, dándose validez a tal efecto a la manifestación de voluntad autonómica que se expresó durante la Segunda República. Pasaban, pues, directamente a la fase de elaboración del Estatuto de Autonomía, que se sustanciaba en un proceso de negociación entre la Asamblea de parlamentarios de la Comunidad Autónoma y la Comisión Constitucional del Congreso de los Diputados, es decir, en una negociación entre la nacionalidad correspondiente y el Estado. La organización política de la Comunidad Autónoma consistiría en una Asamblea legislativa elegida por sufragio universal, un Presidente y un Consejo de Gobierno elegido por dicha Asamblea y un Tribunal Superior de Justicia. El techo competencial sería de manera inmediata el máximo previsto en la Constitución.

El ejercicio del derecho a la autonomía por parte de las nacionalidades desembocaba por tanto en unidades de descentralización del Estado de naturaleza inequívocamente política. Las nacionalidades eran portadoras de un poder político propio, reconocido en la Constitución y subordinado, por tanto, a ella, pero autónomo respecto del poder del Estado.

Con base en tal interpretación de la Constitución se procedió en el primer año de vigencia del texto constitucional a la aprobación de los Estatutos de Autonomía del País Vasco y de Cataluña (LO 3 y 4/1979, de 18 de diciembre) y se tramitaria parlamentariamente el Estatuto de Galicia, que, sin embargo, no sería aprobado de manera definitiva al 
producirse determinadas discrepancias en la negociación del mismo, ante la pretensión del Estado de rebajar en cierta medida el contenido de la autonomía gallega respecto a la vasca y la catalana.

Una vez aprobados los Estatutos de Autonomía del País Vasco y de Cataluña y tramitado parlamentariamente el Estatuto de Galicia, el Gobierno de la Nación tomó la decisión de que todos los demás territorios del Estado, esto es, las regiones, se constituyeran en Comunidades Autónomas por la vía prevista en el artículo 143 de la Constitución, sin precisar en absoluto qué es lo que ello iba a suponer para el ejercicio del derecho a la autonomía de las mencionadas regiones, aunque todo apuntaba hacia una autonomía más administrativa que política, en el sentido del Segundo Anteproyecto de Constitución.

Esto, en todo caso, no lo sabremos nunca, ya que la interpretación nacionalista o diferenciadora de la Constitución tendría que ser abandonada casi inmediatamente, como consecuencia del resultado del referéndum de ratificación de la iniciativa autonómica celebrado en Andalucía el 28 de febrero de 1980.

En Andalucía se había ejercido la iniciativa autonómica en los términos previstos en el artículo 151 de la Constitución, a fin de intentar ejercer el derecho a la autonomía en los mismos términos que las nacionalidades. Se tenía que convocar, en consecuencia, el referéndum de ratificación de tal iniciativa autonómica previsto en el propio artículo 151. Dicho referéndum se celebró, como ha quedado dicho, el 28 de febrero de 1980 y, aunque jurídicamente fracasó, políticamente fue todo un éxito para la voluntad de constituirse en Comunidad Autónoma en las mismas condiciones que las nacionalidades. Hubo, en consecuencia, que diseñar una fórmula para que ello pudiera hacerse. A las tres nacionalidades se sumaba una región en el ejercicio del derecho a la autonomía.

Y no una región cualquiera, sino la región más extensa y más poblada con diferencia de todo el Estado. Si lo que sucedió en Andalucía hubiera sucedido en Cantabria, tal vez se hubiera podido mantener la interpretación diferenciadora de la Constitución, pero con Andalucía no era posible. Andalucia no puede ser configurada políticamente como excepción. La norma andaluza no podía dejar de ser la norma para el ejercicio del derecho a la autonomía de todas las regiones. De ahí que hubiera que proceder a interpretar la Constitución desde la otra perspectiva posible: la de considerar la autonomía como un problema general de la estructura del Estado. 
Tal interpretación se impondría con una rapidez notable, mediante una negociación de naturaleza política entre los dos grandes partidos españoles del momento: UCD, que era el partido que ocupaba el Gobierno de la Nación, y el PSOE, que era, con mucha diferencia, el principal partido de la oposición. Dicha negociación culminaría en unos llamados "Pactos Autonómicos", suscritos en julio de 1981 únicamente por el Gobierno y el PSOE, ya que de su negociación se retirarían progresivamente todos los demás partidos parlamentarios.

En dichos "Pactos Autonómicos" se procedería a concretar la estructura del Estado a partir del texto constitucional, precisándose los siguientes extremos:

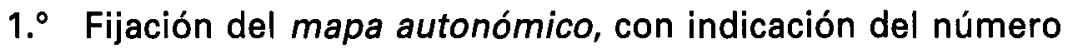
de la futuras Comunidades Autónomas y de las provincias que integrarían cada una de ellas, así como las Comunidades Autónomas uniprovinciales.

2. Definición de todas las Comunidades Autónomas como Comunidades de naturaleza política, con una organización institucional idéntica a las de las nacionalidades, esto es, con Asamblea legislativa, Presidente y Consejo de Gobierno y Tribunal Superior de Justicia.

3. Fijación del plazo en el que se pondría fin al proceso autonómico: 1 de febrero de 1983.

Los «Pactos Autonómicos» serían cumplidos escrupulosamente por los firmantes de los mismos, que disponían de las mayorías parlamentarias suficientes para ello. En sucesivas oleadas, diciembre del 81 (Andalucía, Asturias y Cantabria), junio-agosto del 82 (La Rioja, Murcia, Comunidad Valenciana, Aragón, Castilla-La Mancha, Canarias, Navarra) y febrero del 83 (Extremadura, Baleares, Madrid y Castilla y León) se aprobarían los Estatutos de todas las regiones, que se constituían de esta manera en Comunidades Autónomas.

Los "Pactos Autonómicos" de julio de 1981 serían completados con unos nuevos "Pactos" en febrero de 1982, suscritos nuevamente por los dos grandes partidos españoles exclusivamente, aunque esta vez con el PSOE en el Gobierno y el PP en la oposición. Los demás partidos parlamentarios también se negaron en esta ocasión a suscribir los mencionados "Pactos". En estos últimos se acordaría la ampliación del techo competencial de todas las Comunidades Autónomas que se habían constituido por la vía del artículo 143 de la Constitución hasta equipararlas a las nacionalidades también en esta fase de ejercicio del derecho a la autonomía. 
Así pues, el Estado español, al final de los distintos procesos estatuyentes, es un Estado políticamente descentralizado en su totalidad, en el que todo el territorio está organizado en Comunidades Autónomas que tienen la misma naturaleza, la misma organización política, el mismo techo competencial y la misma financiación. Aunque el proceso ha sido muy embarullado y heterogéneo, el resultado final ha sido extraordinariamente homogéneo.

En realidad, la concreción final de la estructura del Estado ha supuesto de facto un retorno al diseño del primer Anteproyecto de Constitución. El proceso de formación del Estado de las Autonomias ha sido completamente distinto al que se contemplaba en aquel Anteproyecto, pero el resultado final ha sido muy parecido. Podría pensarse, en consecuencia, que, de la misma manera que Dios escribe recto con rasgos torcidos, la sociedad española también habría dado respuesta, de manera embarullada, pero respuesta, a su principal problema constituyente. La estructura del Estado finalmente impuesta a partir de la Constitución y con base en ella no es una estructura parcial, sino una estructura general y uniforme del Estado.

$Y$ sin embargo no es así. Si el resultado se hubiera alcanzado a partir del primer Anteproyecto de Constitución, se habría debatido y decidido en sede constituyente la estructura del Estado. Se habría debatido y decidido por todos los partidos representados en las Cortes Constituyentes la estructura que se estaba dando al Estado. Nadie habría quedado excluido del debate y decisión. Y además el pueblo español, en el referéndum de ratificación del texto aprobado por las Cortes Constituyentes, hubiera tenido que pronunciarse expresamente y dar su asentimiento a la estructura del Estado constitucionalmente definida.

Con una discusión pública y solemne en sede constituyente, esto es, con la participación de todos los partidos con representación parlamentaria y con la prestación del consentimiento de todos ellos, así como con la prestación del consentimiento de los ciudadanos a través de referéndum, se hubiera podido dar una respuesta constitucional clara e inequívoca a la estructura del Estado, que difícilmente habría podido ser legítimamente cuestionada con posterioridad.

La estructura final del Estado se acabó definiendo sin debate general sobre la misma, a través de unos "Pactos Autonómicos", en los que el consenso fue mucho menor que en la elaboración del texto constitucional y sin participación directa del cuerpo electoral.

Dicho en pocas palabras: en la respuesta al problema constituyente más importante de la sociedad española han faltado o, mejor 
dicho, han estado presentes de manera sumamente imperfecta, todos los elementos que tienen que estar presentes en una respuesta constituyente: debate general transparente, consenso prácticamente unánime y participación directa del cuerpo electoral. De ahí que el problema no esté resuelto, aunque tampoco está sin resolver.

Y no está sin resolver porque el Estado español tiene una estructura territorial jurídica definida y cerrada. No sólo en la Constitución, pero sí a través de la Constitución y los diecisiete Estatutos de Autonomia, que constituyen lo que en la doctrina española, haciendo uso de una terminología de origen francés, se denomina el «bloque de la constitucionalidad». Tanto la Constitución como los Estatutos de Autonomía son normas rígidas, que disponen de procedimientos de reforma distintos del procedimiento legislativo ordinario y, en consecuencia, la estructura del Estado está cerrada como pueden estar cerradas las normas de naturaleza constitucional.

El Estado español no sólo tiene, pues, una estructura territorial protegida a través de la técnica exclusivamente constitucional de la reforma, constitucional y estatutaria, sino que se trata, además, de una estructura que funciona. Desde 1980, en que se celebraron las primeras elecciones autonómicas en el País Vasco y Cataluña, hasta hoy, se han celebrado elecciones legislativas en todas las Comunidades Autónomas en al menos cinco ocasiones, estando, en consecuencia, contrastado en la práctica el diseño final de la estructura del Estado. La forma del Estado de las Autonomías es una forma estatal realmente existente, cuya legitimidad de ejercicio, por utilizar la terminología escolástica clásica, resulta difícilmente discutible. Ha dado respuesta de una manera razonablemente eficaz al problema de la articulación territorial del Estado, transformando a uno de los Estados más unitarios y centralistas del mundo en uno de los más descentralizados. $Y$ de una manera inequívocamente democrática, a través de procesos electorales libres y competidos.

Y sin embargo, el problema dista de estar resuelto. El déficit de legitimidad de origen no ha podido ser compensado por el funcionamiento en la práctica del Estado de las Autonomías. La estructura territorial del Estado ha sido definida democráticamente. Los partidos que firmaron los "Pactos Autonómicos" de 1981 y 1992 representaban más del $80 \%$ de la población de la sociedad española y disponían de más del $80 \%$ de los escaños en las Cortes. Desde el punto de vista de la legalidad no se le puede poner el más mínimo reparo a su manera de proceder. Tenían derecho a hacer lo que hicieron y no decidieron nada inconfesable, que no hubiera podido hacerse a través de un debate 
público. Pero, a pesar de ello, ha habido un déficit de legitimidad en la decisión final a través de la cual se acabó concretando la estructura del Estado. $Y$ mientras dicho déficit de legitimidad no sea eliminado, el problema no estará definitivamente resuelto.

Dicho déficit de legitimidad sólo puede ser suprimido mediante la reforma de la Constitución. Únicamente mediante el ejercicio del poder constituyente constituido se podría subsanar el que podríamos denominar pecado original de la definición de nuestra estructura del Estado.

Ciertamente la reforma de la Constitución que se hiciera tendría que tener un alcance limitado. La estructura del Estado ya está definida y está operando, y no es imaginable que se pudiera volver otra vez a intentar diferenciar las nacionalidades de las regiones, como se hizo en el tránsito del primer al segundo Anteproyecto de Constitución. Si eso no se pudo hacer ni siquiera en la fase de inicial puesta en marcha de la Constitución, mucho menos se podría hacer en estos momentos.

Pero sí se podría proceder a la reforma del Senado y, con ocasión de ella, abrir un debate sobre la articulación de todas las Comunidades Autónomas en el órgano constitucional representativo del pueblo español (art. 66.1 CE).

El Senado es un órgano extraordinariamente mal constituido. EI constituyente, como hemos visto, no definió la estructura del Estado $y$, en consecuencia, no fue tampoco capaz de definir el órgano constitucional en el que la estructura descentralizada del Estado tendría que encontrar su reflejo. El Senado quedó definido como un Senado provincial con el añadido de algunos senadores autonómicos. Es una Cámara que carece de sentido en los términos en que figura en la Constitución, existiendo una contradicción patente entre su definición como Cámara territorial en el apartado 1 del artículo 69 y su composición establecida en los apartados siguientes del mismo artículo. La composición del Senado es la huella que queda en el texto constitucional del déficit de legitimidad de origen en la definición de la estructura del Estado. Mientras esa huella no desaparezca, el déficit estará marcando nuestra vida política.

El Senado es en buena medida el problema y la solución. Es el problema por lo que supone de recuerdo del déficit de legitimidad y porque es un obstáculo a la integración armónica de las Comunidades Autónomas en el Estado. Es la solución, porque en el debate y la decisión sobre su reforma se puede acabar poniendo fin al mencionado 
déficit de legitimidad y se pueden diseñar unas fórmulas a través de las cuales las Comunidades Autónomas se relacionen entre sí y con el Estado de una manera coherente con lo que realmente son. Las Comunidades Autónomas, como los personajes de Pirandello, están buscando un autor, que les permita que su vida real, que es riquísima, encuentre el escenario apropiado, que no puede ser otro que el texto constitucional.

La reforma del Senado está en la agenda política desde hace ya algunos años, aunque no es probable que, mientras el PP se mantenga en el poder, se vaya a proceder a la misma. Es más que probable que, durante algún tiempo, continuemos hablando en prosa sin saberlo y continuemos negándonos a hablar abiertamente del problema de cómo los distintos pueblos y territorios de España tienen que organizar su convivencia en una casa común, en un único Estado. 